\section{The most reliable black hole yet}

\author{
from M.G. Watson
}

BL.ACK holes are frequently invoked by astronomers to explain the vast amount of energy radiated from the nuclei of many galaxies. The energy is produced, in this picture, as material surrounding the black hole falls down its extremely steep gravitational potential well. The same phenomenon is believed to occur on a much smaller scale in a few X-ray-emitting binary stars in or near our Galaxy, providing the best available evidence that these theoretically predicted objects exist at all. In these X-ray binaries the infalling matter eventually accreted by the black hole is supplied by the companion star. A recent investigation of a system of this type - an $\mathrm{X}$-ray transient - has produced results which arguably place the existence of black holes on considerably firmer ground (McClintock, J.E. \& Remillard, R.A. Astrophys. J. in the press).

$\mathrm{X}$-ray transients are a small but important subclass of X-ray binaries. Most are believed to be systems containing a faint red star and an accreting compact object (a neutron star or black hole). As such they are closely similar to the persistent low-mass X-ray binaries (White, N.E. \& Mason, K.O. Space Sci. Rev. 40, 167; 1985), the principal difference being that $X$-ray transients are only luminous X-ray emitters during their relatively short (of the order of months) outbursts which recur on timescales of years, a pattern of behaviour which presumably reflects a highly variable mass transfer rate onto the compact object. The X-ray transient $\mathrm{A}(0620-00$ is perhaps the most famous member of its class as it was, for a few months in 1975, the brightest celestial Xray source ever recorded (Elvis, $\mathrm{M}$ et al. Nature 257, 656; 1975). Despite the enormous observational attention that it attracted then, little was learnt about the properties of the underlying binary system. A decade later, the new detailed optical study of the A0620-00 system (now in its quiescent state) by McClintock and Remillard provides compelling evidence that the binary system contains a black hole.

McClintock and Remillard's result is based on several years of photometric observation of $\mathrm{A} 0620-00$ in quiescence that provide a unique orbital period of 7.75 , together with just one night's spectroscopy which allowed them to determine the radial velocity curve of the faint red companion to the compact object by measuring the periodic Doppler shifts of its absorption lines. The amplitude of the radial velocity curve, $457 \pm 8 \mathrm{~km} \mathrm{~s}^{-1}$, is very large for a binary with such a short period, and this measurement alone sets a firm lower limit to the mass of the compact cantly above the maximum mass for a neutron star for the stiffest equation of state of neutron matter considered realistic, leading inexorably to the conclusion that the compact object must be a black hole. A more realistic estimate of the compact object mass can be made by assuming a reasonable mass for the red companion star, and using the constraints on the orbital inclination placed by the lack of Xray eclipses and the modelling of the photometric light curve. On this basis McClintock and Remillard conclude that the mass of the compact object is likely to be greater than 7 solar masses.

The X-ray transient A0620-00 thus joins $\mathrm{CygX}-1$ and two systems in the Large Magellanic Cloud, LMC X-1 and LMC X-3, as the fourth system which is a good candidate for a black-hole binary on the basis of dynamical measurements of the compact object mass. In contrast to the other three black-hole binaries which have hot, luminous, massive companions, the companion in A0620-00 is clearly a faint, low-mass red star. The presence of a massive black hole in this system may pose a fresh set of evolutionary questions. In the black hole could have formed in this system without the binary being totally disrupted. The mass of the progenitor star would have had to be at least 20 solar masses (probably more), hence it seems very likely that the formation of the black hole would have involved the loss of more than half the system mass, leading to the binary becoming unbound. The present short binary period is also difficult to reconcile with the size of the original binary system required to accommodate the large, massive progenitor of the present black hole. The difficulties associated with understanding the evolutionary history of A0620-00 suggest that it is a rather rare system. It is too early to say whether this is indeed the case, because the binary system parameters of a large fraction of all Xray binaries, transient or otherwise, have yet to be measured, but it is interesting to note that the empirical X-ray spectral classification scheme proposed by White and F.E. Marshall (Astrophys. J. 281, 354; 1984) suggested several new black-hole candidates, one of which was A0620-00.

In the case of $A 0620-00$ there can be little doubt about either the correctness of the radial velocity measurements or their interpretation. One of the standard sceptiobject of 3.2 solar masses. This is signifiparticular it is difficult to understand how cal responses to any unlikely dynamical measurement has been to suggest that the system is a triple, rather than a binary. This argument cannot be applied to A0620-00, given the small size of the system implied by the 8 -hour period. Thus, A0620-00 is arguably the most reliable (or the least unreliable) black-hole candidate yet discovered. Because of this, and because it is a nearby galactic system, it may be that the impact of this result will be mainly psychological in making the possibility, if not the reality, of black holes of stellar mass seem more appealing to many astronomers.

M.G. Watson is in the X-ray Astronomy Group, Department of Physics, University of Leicester, Leicester LEI 7RH, UK.

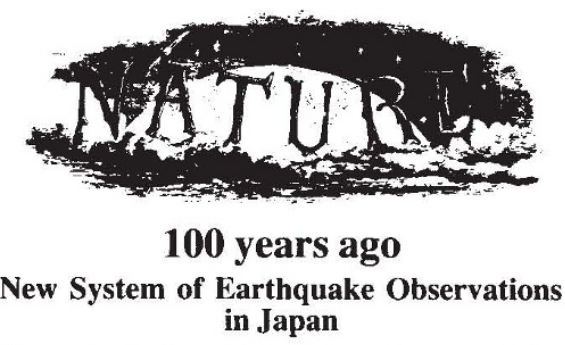

OwING to the invention of new seismographs by the members of the Seismological Society of Japan, there has been of late a complete change in the system of earthquake observations in this country. The Meteorological Bureau now employs the horizontal pendulum and verticalmotion seismographs of Profs. J. Milne and T. Gray, and of Prof. J.A. Ewing for systematic observation, while the Imperial University of Tokio publishes from time to time detailed accounts of particular and more interesting shocks by the use of similar instruments. These seismographs register on a revolving glass plate or drum automatically started by the earthquake motion, components of horizontal and vertical motions of the earth on a magnified scale, thus producing continuous diagrams, and indicating successive displacement of the ground in conjuction with the time. The account of the earthquake of December 28, 1885, the largest shock during the last three months, is here given as a sample of seismic record now issued in this country. The motion is slowly commenced, not accompanied by quick tremors, as is usually the case. At the 14th second from the commencement a considerable E. -W. motion occurred; in another second the maximum movement appeared in the same direction, which was followed by smaller shocks during about one minute; and from thence the oscillations, gradually subsided. As usual, the particles of the ground did not move to and fro, but traced a curvilinear path, although the E. $-W$. components always remained greater than the S. $-\mathrm{N}$. components. In all, over 130 shocks or complete waves were registered. I may add in respect to the above earthquake, and in general, that the vertical motions are always in our experience - smaller than horizontal ones, and the maxima and minima of these two kinds of motions are not synchronous. I shall have occasion before long to communicate to you the general results of all observations made during past years.

The Imperial University of Tokio, Japan.

From Nature 33, 603; 29 April 1886. 